\title{
Stochastic Modeling of a Computer System with Priority to PM over S/W Replacement Subject to Maximum Operation and Repair Times
}

\author{
Ashish Kumar \\ Department of Statistics \\ M.D.University, Rohtak-124001 \\ Haryana (India)
}

\author{
S.C. Malik \\ Department of Statistics \\ M.D.University,Rohtak-124001 \\ Haryana (India)
}

\begin{abstract}
This paper focuses on the stochastic modeling of a computer system of two identical units- one is initially operative and other is kept as spare in cold standby. In each unit $\mathrm{h} / \mathrm{w}$ and s/w components work together and fail independently. There is a single server who visits the system immediately as and when required. The server takes the unit under preventive maintenance after a maximum operation time at normal mode. The $\mathrm{h} / \mathrm{w}$ components under go for repair at their failure and are replaced by new one in case these are not repaired up to a maximum repair time. However, s/w components are replaced by new one instead of repair. Priority is given to the preventive maintenance (PM) of the unit over replacement of the $\mathrm{s} / \mathrm{w}$ components. The failure time distribution of the components follow negative exponential whereas the distributions of preventive maintenance, repair and replacement time are taken as arbitrary with different probability density functions. Several reliability and economic indices have been obtained using semi-Markov and regenerative point technique. The graphical study of the results has also been made.
\end{abstract}

\section{General Terms}

Stochastic Process

\section{Keywords}

Computer System, Stochastic Model, Preventive Maintenance, Maximum Operation and Repair Times, Priority, Replacement, Reliability and Economic measures.

\section{INTRODUCTION}

The remarkable progress in the field of computer technology has resulted in the widespread usage of computer applications in almost all academic, business and industrial sectors. A major challenge to the industrialists now a day is to provide reliable $\mathrm{h} / \mathrm{w}$ and $\mathrm{s} / \mathrm{w}$ components. Most of the academicians are also trying to explore new techniques for reliability improvement of the computer systems. In spite of these efforts, a little work has been dedicated to the reliability modeling of computer systems. And, most of the research work carried out so far in the subject of s/w and h/w reliability has been limited to the consideration of either $\mathrm{h} / \mathrm{w}$ subsystem alone or $\mathrm{s} / \mathrm{w}$ subsystem alone. But there are many complex systems in which $\mathrm{h} / \mathrm{w}$ and $\mathrm{s} / \mathrm{w}$ components work together to provide computer functionality. Friedman and Tran [1] and Welke et al.[2] tried to establish a combined reliability model for the whole system in which hardware and software components work together. Recently, Malik and Anand $[4,7,8]$ and Malik and kumar [6] suggested reliability models of a computer system with independent failure of $h / w$ and $s / w$ components.

Further, the continued operation and ageing of these systems gradually reduce their performance, reliability and safety. And, a breakdown of such systems is costly, dangerous and may create confusion in our society. It is, therefore, of great importance to operate such systems with high reliability. It is proved that preventive maintenance can slow the deterioration process of a repairable system and restore the system in a younger age or state. Thus, the method of preventive maintenance can be used to improve the reliability and profit of system. Malik and Nandal [5] has proposed a reliability model for complex systems introducing the concept of preventive maintenance of the unit after a maximum operation time. Further, the reliability of a system can be increased by making replacement of the components by new one in case repair time is too long i.e., if it extends to a pre-specific time. Singh and Agrafiotis[3] analyzed stochastically a two-unit cold standby system subject to maximum operation and repair time.

In view of the above and considering the practical importance of computer systems in our daily lives, a stochastic model of a computer system of two identical units- one is initially operative and other is kept as spare in cold standby is developed. In each unit $\mathrm{h} / \mathrm{w}$ and $\mathrm{s} / \mathrm{w}$ components work together and fail independently. A single server is provided immediately to the system as and when required. The server takes the unit under preventive maintenance after a maximum operation time at normal mode. The $\mathrm{h} / \mathrm{w}$ components under go for repair at their failure and are replaced by new one in case these are not repaired up to a maximum repair time. However, s/w components are replaced by new one instead of repair. Priority is given to the preventive maintenance (PM) of the unit over replacement of the s/w components. The failure time distribution of the components follow negative exponential whereas the distributions of preventive maintenance, repair and replacement time are taken as arbitrary with different probability density functions. Some reliability and economic measures of the system model such as mean time to system failure (MTSF), availability, busy period of the server due to $\mathrm{PM}$, busy period of the server due to $\mathrm{h} / \mathrm{w}$ repair, busy period of the server due to $\mathrm{h} / \mathrm{w}$ replacement, busy period of the server due to $\mathrm{s} / \mathrm{w}$ replacement, expected number of $\mathrm{h} / \mathrm{w}$ replacements, expected number of s/w replacements, expected number of visits of the server and profit function are obtained using semi-Markov and regenerative point technique. The graphical behaviour of the results has also been shown for a particular case to make the study more effective. 


\section{NOTATIONS}

$\begin{array}{lll}\mathrm{E} & : & \text { The set of regenerative states } \\ \mathrm{NO} & : & \text { The unit is operative and in normal mode } \\ \mathrm{Cs} & : & \text { The unit is in cold standby } \\ \mathrm{a} / \mathrm{b} & : & \begin{array}{l}\text { Probability that the system has hardware / } \\ \text { software failure }\end{array} \\ \lambda_{1} / \lambda_{2} & : & \begin{array}{c}\text { Constant hardware / software failure rate } \\ \text { Maximum constant rate of Operation }\end{array}\end{array}$

$\alpha_{0}$

Time

\begin{tabular}{|c|c|c|}
\hline $\begin{array}{l}\beta_{0} \\
\mathrm{Pm} / \mathrm{PM}\end{array}$ & : & $\begin{array}{l}\text { Maximum constant rate of Repair Time. } \\
\text { The unit is under preventive Maintenance/ }\end{array}$ \\
\hline
\end{tabular}

under

preventive maintenance continuously from previous state

WPm/WPM : The unit is waiting for PM / waiting for preventive

state

HFur/HFUR maintenance continuously from previous is
The unit is failed due to hardware and
under repair / under repair continuously from previous state

HFurp/HFURP : $\quad$ The unit is failed due to $h / w$ and is under

replacement / under replacement continuously from previous state

HFwr / HFWR : $\quad$ The unit is failed due to $\mathrm{h} / \mathrm{w}$ and is waiting for

repair/waiting for repair continuously from previous state

SFurp/SFURP :

The unit is failed due to the s/w and is under

SFwrp/SFWRP

replacement/under

replacement

continuously from previous state

and is

$\mathrm{h}(\mathrm{t}) / \mathrm{H}(\mathrm{t})$

of unit due to

$\mathrm{g}(\mathrm{t}) / \mathrm{G}(\mathrm{t})$

hardware

$\mathrm{m}(\mathrm{t}) / \mathrm{M}(\mathrm{t})$

hardware

$\mathrm{f}(\mathrm{t}) / \mathrm{F}(\mathrm{t})$

$\mathrm{q}_{\mathrm{ij}}(\mathrm{t}) / \mathrm{Q}_{\mathrm{ij}}(\mathrm{t})$

$\mathrm{df} / \mathrm{cdf}$

Cumulative density

$\mathrm{q}_{\mathrm{ij} . k r}(\mathrm{t}) / \mathrm{Q}_{\mathrm{ij} . k r}(\mathrm{t})$ : regenerative

$\mu_{\mathrm{i}}(\mathrm{t})$
The unit is failed due to the software

waiting for replacement / waiting for replacement continuously from previous state

pdf / cdf of replacement time

software

pdf / cdf of repair time of the

pdf / cdf of replacement time of the

pdf / cdf of the time for PM of the unit

pdf / cdf of passage time from

regenerative state $\mathrm{i}$ to a regenerative state $\mathrm{j}$ or to a failed state $\mathrm{j}$ without visiting any other regenerative state in $(0, \mathrm{t}]$

Probability density function/

function

pdf/cdf of direct transition time from

state $\mathrm{i}$ to a regenerative state $\mathrm{j}$ or to a failed state $\mathrm{j}$ visiting state $\mathrm{k}, \mathrm{r}$ once in $(0$, t]

Probability that the system up initially in state $S_{i} \in E$ is up at time $t$ without visiting to any regenerative state
$\mathrm{W}_{\mathrm{i}}(\mathrm{t})$ state $S_{\mathrm{i}}$ upto time ' $\mathrm{t}$ 'without making any transition to any other regenerative state or returning to the same state via one or more non-regenerative states.

$m_{i j}$

Contribution to mean sojourn time $\left(\mu_{\mathrm{i}}\right)$ in state $S_{i}$ when system transit directly to state $S_{j}$ so that

$$
\mu_{i}=\sum_{j} m_{i j} \text { and } \mathrm{m}_{\mathrm{ij}}=\int t d Q_{i j}(t)=-q_{i j}^{*{ }^{\prime}}(0)
$$

(8) convolution/Laplace

Symbol for Laplace-Stieltjes

convolution

$\sim$ / : Symbol for Laplace Steiltjes Transform (LST) /

Laplace Transform (LT)

'(desh) : Used to represent alternative result

\section{RELIABILITY INDICES}

\subsection{Transition Probabilities And Mean}

Sojourn Times

Simple probabilistic considerations yield the following expressions for the non-zero elements

$p_{i j}=Q_{i j}(\infty)=\int_{0}^{\infty} q_{i j}(t) d t \quad$ using the notations $\mathrm{A}=$ $\mathrm{a} \lambda_{1}+\mathrm{b} \lambda_{2}+\alpha_{0}$, and $\mathrm{B}=\mathrm{a} \lambda_{1}+\mathrm{b} \lambda_{2}+\alpha_{0}+\beta_{0}$ as

(1)

$$
\begin{aligned}
& \mathrm{p}_{01}=\frac{\alpha 0}{A}, \mathrm{p}_{02}=\frac{a \lambda 1}{A}, \mathrm{p}_{03}=\frac{b \lambda_{2}}{A}, \mathrm{p}_{10}=f^{*}(\mathrm{~A}), \mathrm{p}_{16}=\frac{a \lambda 1}{A}[1-f \\
& \left.{ }^{*}(\mathrm{~A})\right]=\mathrm{p}_{12.6}, \mathrm{p}_{18}=\frac{b \lambda_{2}}{A}\left[1-f^{*}(\mathrm{~A})\right]=\mathrm{p}_{13.8,} \mathrm{p}_{1.13}=\frac{\alpha 0}{A}[1-f \\
& \left.{ }^{*}(\mathrm{~A})\right]=\mathrm{p}_{11.13}, \quad \mathrm{p}_{20}=g^{*}(\mathrm{~B}), \mathrm{p}_{24}=\frac{\beta_{0}}{B}\left[1-g^{*}(\mathrm{~B})\right], \mathrm{p}_{25}=\frac{\alpha 0}{B}[
\end{aligned}
$$$$
\left.1-g^{*}(\mathrm{~B})\right] \mathrm{p}_{2.11}=\frac{b \lambda 2}{B}\left[1-g^{*}(\mathrm{~B})\right], \mathrm{p}_{2.12}=\frac{a \lambda 1}{B}\left[1-g^{*}(\mathrm{~B})\right], \mathrm{p}_{30}=
$$$$
h^{*}(\mathrm{~A}), \mathrm{p}_{37}=\frac{a \lambda 1}{A}\left[1-h^{*}(\mathrm{~A})\right]=\mathrm{p}_{32.7}, \mathrm{p}_{39}=\frac{\alpha_{0}}{A}\left[1-h^{*}(\mathrm{~A})\right],
$$$$
\mathrm{p}_{40}=m^{*}(\mathrm{~A}), \mathrm{p}_{3,10}=\frac{b \lambda_{2}}{A}\left[1-h^{*}(\mathrm{~A})\right]=\mathrm{p}_{33.10}, \quad \mathrm{p}_{51}=g^{*}\left(\beta_{0}\right),
$$$$
\mathrm{p}_{5,16}=1-g^{*}\left(\beta_{0}\right), \quad \mathrm{p}_{4.17}=\frac{\alpha 0}{A}\left[1-m^{*}(\mathrm{~A})\right]=\mathrm{p}_{41.17,} \mathrm{p}_{62}=f^{*}(0),
$$$$
\mathrm{p}_{72}=h^{*}(0), \mathrm{p}_{83}=f^{*}(0), \quad \mathrm{p}_{93}=f^{*}(0), \quad \mathrm{p}_{10.3}=h^{*}(0), \quad \mathrm{p}_{11.3}=g
$$$$
{ }^{*}\left(\beta_{0}\right), \quad \mathrm{p}_{11.14}=1-g^{*}\left(\beta_{0}\right), \mathrm{p}_{4,18}=\frac{b \lambda_{2}}{A}\left[1-m^{*}(\mathrm{~A})\right]=\mathrm{p}_{43.18}, \mathrm{p}_{12.2}
$$$$
=g^{*}\left(\beta_{0}\right), \quad \mathrm{p}_{12.15}=1-g^{*}\left(\beta_{0}\right), \mathrm{p}_{13.1}=f^{*}(0), \quad \mathrm{p}_{14.3}=
$$$$
m^{*}(0), \mathrm{p}_{4.19}=\frac{a \lambda 1}{A}\left[1-m^{*}(\mathrm{~A})\right]=\mathrm{p}_{42.19,} \mathrm{p}_{15.2}=m^{*}(0), \quad \mathrm{p}_{16.1}=
$$$$
m^{*}(0), \mathrm{p}_{17.1}=m^{*}(0), \mathrm{p}_{18.3}=m^{*}(0), \mathrm{p}_{19.2}=m^{*}(0), \mathrm{p}_{21.5}=\frac{\alpha_{0}}{B}[1-
$$ 
$\left.g^{*}(\mathrm{~B})\right] g^{*}\left(\beta_{0}\right), \mathrm{p}_{21.5,16}=\frac{\alpha 0}{B}\left[1-g^{*}(\mathrm{~B})\right]\left[1-g^{*}\left(\beta_{0}\right)\right], \mathrm{p}_{23.11}=$ $\frac{b \lambda_{2}}{B}\left[1-g^{*}(\mathrm{~B})\right]\left[g^{*}\left(\beta_{0}\right)\right], \mathrm{p}_{23.11,14}=\frac{b \lambda_{2}}{B}\left[1-g^{*}(\mathrm{~B})\right][1-g$ $\left.{ }^{*}\left(\beta_{0}\right)\right], \mathrm{p}_{22.12}=\frac{a \lambda 1}{B}\left[1-g^{*}(\mathrm{~B})\right] g^{*}\left(\beta_{0}\right), \mathrm{p}_{22.12,15}=\frac{a \lambda 1}{B}[1-$ $\left.g^{*}(\mathrm{~B})\right]\left[1-g^{*}\left(\beta_{0}\right)\right]$

It can be easily verified that $\mathrm{p}_{01}+\mathrm{p}_{02}+\mathrm{p}_{03}=\mathrm{p}_{10}+\mathrm{p}_{16}+\mathrm{p}_{18}+\mathrm{p}_{1.13}=$ $\mathrm{p}_{20}+\mathrm{p}_{24}+\mathrm{p}_{25}+\mathrm{p}_{2,11}+\mathrm{p}_{2.12}=\mathrm{p}_{30}+\mathrm{p}_{37}+\mathrm{p}_{39}+\mathrm{p}_{3,10}=\mathrm{p}_{40}+\mathrm{p}_{4.17}+\mathrm{p}_{4.18}+$ $\mathrm{p}_{4.19}=\mathrm{p}_{5.1}+\mathrm{p}_{5.16}=\mathrm{p}_{62}=\mathrm{p}_{72}=\mathrm{p}_{83}=\mathrm{p}_{91}=\mathrm{p}_{10.3}=\mathrm{p}_{11.3}+\mathrm{p}_{11.14}=$ $\mathrm{p}_{12.2}+\mathrm{p}_{12.15}=\mathrm{p}_{13.1}=\mathrm{p}_{14.1}=\mathrm{p}_{15.2}=\mathrm{p}_{16.1}=\mathrm{p}_{17.1}=\mathrm{p}_{18.3}=\mathrm{p}_{19.2}=$ $\mathrm{p}_{10}+\mathrm{p}_{12.6}+\mathrm{p}_{11.13}+\mathrm{p}_{13.8}=\mathrm{p}_{20}+\mathrm{p}_{24}+\mathrm{p}_{21.5}+\mathrm{p}_{21.5,16}+\mathrm{p}_{23,11}+\mathrm{p}_{23.11,14}$ $+\mathrm{p}_{22,12}+\mathrm{p}_{22.12,15}=\mathrm{p}_{30}+\mathrm{p}_{31.9}+\mathrm{p}_{32.7}+\mathrm{p}_{33.10}=\mathrm{p}_{40}+\mathrm{p}_{41.17}+\mathrm{p}_{42.19}+$ $\mathrm{p}_{43.18}=1$

The mean sojourn times $\left(\mu_{\mathrm{i}}\right)$ is the state $\mathrm{S}_{\mathrm{i}}$ are

$\mu_{0}=\frac{1}{A} \mu_{1}=\frac{1}{A+\alpha}, \mu_{2}=\frac{1}{\theta+B}, \mu_{3}=\frac{1}{A+\beta}, \quad \mu_{4}=\frac{1}{A+\gamma}, \mu_{1}^{\prime}$ $=\frac{1}{\alpha}, \mu_{3}^{\prime}=\frac{1}{\beta}, \mu_{4}^{\prime}=\frac{1}{\gamma}$,

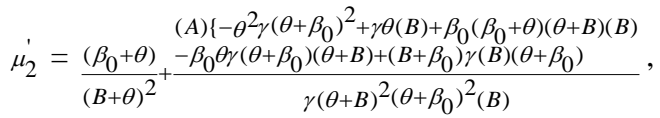

\subsection{Mean Time to System Failure}

Let $\phi_{\mathrm{i}}(\mathrm{t})$ be the c.d.f of first passage time from the regenerative state $i$ to a failed state. Regarding the failed state as absorbing state, we have the following recursive relation for $\phi_{\mathrm{i}}(\mathrm{t})$ :

$$
\phi_{\mathrm{i}}(\mathrm{t})=\sum_{\mathrm{j}} \mathrm{Q}_{\mathrm{i}, \mathrm{j}}(\mathrm{t}) \circledast \phi_{\mathrm{j}}(\mathrm{t})+\sum_{\mathrm{k}} \mathrm{Q}_{\mathrm{i}, \mathrm{k}}(\mathrm{t})
$$

Where $j$ is an un-failed regenerative state to which the given regenerative state $i$ can transit and $k$ is a failed state to which the state $i$ can transit directly. Taking LT of above relation (6) and solving for $\tilde{\phi}_{0}(s)$

We have $\mathrm{R} *(\mathrm{~s})=\frac{1-\tilde{\varphi}_{0}(s)}{s}$

The reliability of the system model can be obtained by taking Laplace inverse transform of (7). The mean time to system failure (MTSF) is given by $\mathrm{MTSF}=\lim _{s \rightarrow o} \frac{1-\tilde{\phi}_{0}(s)}{s}=\frac{N_{1}}{D_{1}}$ where

$\mathrm{N}_{1}=\mu_{0}+p_{01} \mu_{1}+p_{02} \mu_{2}+p_{03} \mu_{3}+p_{24} p_{02} \mu_{4}$ and $\mathrm{D}_{1}=$ $1-p_{01} p_{10}-p_{02} p_{20}-p_{03} p_{30}-p_{02} p_{24} p_{40}$

\subsection{Steady State Availability}

Let $A_{i}(t)$ be the probability that the system is in upstate at instant ' $t$ ' given that the system entered regenerative state $\mathrm{i}$ at $\mathrm{t}=0$. The recursive relations for $\mathrm{A}_{\mathrm{i}}(\mathrm{t})$ are given as

$$
A_{i}(t)=M_{i}(t)+\sum_{j} q_{i, j}^{(n)}(t) \Subset A_{j}(t)
$$

Where $j$ is any successive regenerative state to which the regenerative state $i$ can transit through

$\mathrm{n} \geq 1$ (natural number) transitions. $\mathrm{M}_{\mathrm{i}}(\mathrm{t})$ is the probability that the system is up initially in state $S_{i} \in E$ is up at time $\mathrm{t}$ without visiting to any other regenerative state, we have

$$
\begin{aligned}
& M_{0}(t)=e^{-\left(a \lambda_{1}+b \lambda_{2}+\alpha_{0}\right) t}, M_{1}(t)=e^{-\left(a \lambda_{1}+b \lambda_{2}+\alpha_{0}\right) t} \overline{F(t)}, \\
& M_{2}(t)=e^{-\left(a \lambda_{1}+b \lambda_{2}+\alpha_{0}+\beta_{0}\right) t} \overline{G(t)}, \quad M_{3}(t)=e^{-\left(a \lambda_{1}+b \lambda_{2}+\alpha_{0}\right) t} \overline{H(t)}, \\
& M_{4}(t)=e^{-\left(a \lambda_{1}+b \lambda_{2}+\alpha_{0}\right) t} \overline{M(t)}
\end{aligned}
$$

Taking LT of above relations (9) and solving for $A_{0}^{*}(s)$, the steady state availability is given by

$$
A_{0}(\infty)=\lim _{s \rightarrow 0} s A_{0}^{*}(s)=\frac{N_{2}}{D_{2}}, \text { where }
$$

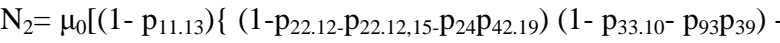
$\left.\mathrm{p}_{32.7}\left(\mathrm{p}_{23.11}+\mathrm{p}_{23.11,14}+\mathrm{p}_{24} \mathrm{p}_{43.18}\right)\right\}-\mathrm{p}_{12.6}\left\{\left(\mathrm{p}_{21.5}+\mathrm{p}_{21.5,16}+\mathrm{p}_{24} \mathrm{p}_{41.17}\right)\right.$ (1- $\left.\left.\mathrm{p}_{33.10^{-}} \mathrm{p}_{93} \mathrm{p}_{39}\right)-\mathrm{p}_{13.8} \mathrm{p}_{32.7}\left\{\left(\mathrm{p}_{21.5}+\mathrm{p}_{21.5,16}+\mathrm{p}_{24} \mathrm{p}_{41.17}\right)\right\}\right]+$ $\mu_{1}\left[\left(\mathrm{p}_{01}\right)\left\{\quad\left(1-\mathrm{p}_{22.12} \mathrm{p}_{\left.22.12,15-\mathrm{p}_{24} \mathrm{p}_{42.19}\right) \quad\left(1-\mathrm{p}_{33.10^{-}}\right.} \mathrm{p}_{93} \mathrm{p}_{39}\right)\right.\right.$ $\left.\mathrm{p}_{32.7}\left(\mathrm{p}_{23.11}+\mathrm{p}_{23.11,14}+\mathrm{p}_{24} \mathrm{p}_{43.18}\right)\right\}+\mathrm{p}_{02}\left\{\left(\mathrm{p}_{21.5}+\mathrm{p}_{21.5,16}+\mathrm{p}_{24} \mathrm{p}_{41.17}\right)\right.$ $\left.\left(1-\mathrm{p}_{33.10^{-}} \mathrm{p}_{93} \mathrm{p}_{39}\right)+\mathrm{p}_{03} \mathrm{p}_{32.7}\left\{\left(\mathrm{p}_{21.5}+\mathrm{p}_{21.5,16}+\mathrm{p}_{24} \mathrm{p}_{41.17}\right)\right\}\right]+\left(\mu_{2}+\right.$ $\left.\mathrm{p}_{24} \mu_{4}\right)\left[\mathrm{p}_{32.7}\left\{\mathrm{p}_{01} \mathrm{p}_{13.8}+\mathrm{p}_{03}\left(1-\mathrm{p}_{11.13}\right)\right\}+\left(1-\mathrm{p}_{33.10^{-}} \mathrm{p}_{93} \mathrm{p}_{39}\right)\left\{\mathrm{p}_{01}\right.\right.$ $\left.\left.\mathrm{p}_{12.6}+\left(1-\mathrm{p}_{11.13}\right) \mathrm{p}_{02}\right\}\right]+\mu_{3}\left[\mathrm{p}_{01}\left\{\mathrm{p}_{12.6}\left(\mathrm{p}_{23.11}+\mathrm{p}_{23.11,14}+\mathrm{p}_{24} \mathrm{p}_{43.18}\right)+\right.\right.$

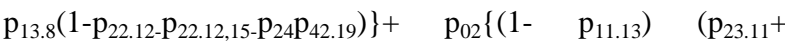
$\left.\left.\mathrm{p}_{23.11,14}+\mathrm{p}_{24} \mathrm{p}_{43.18}\right)+\mathrm{p}_{13.8}\left(\mathrm{p}_{21.5}+\mathrm{p}_{21.5,16}+\mathrm{p}_{24} \mathrm{p}_{41.17}\right)\right\}+\mathrm{p}_{03}\{(1-$

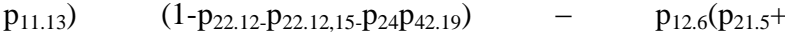
$\left.\left.\left.\mathrm{p}_{21.5,16}+\mathrm{p}_{24} \mathrm{p}_{41.17}\right)\right\}\right]$ 
and

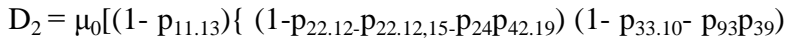
- $\left.\mathrm{p}_{32.7}\left(\mathrm{p}_{23.11}+\mathrm{p}_{23.11,14}+\mathrm{p}_{24} \mathrm{p}_{43.18}\right)\right\}-\mathrm{p}_{12.6}\left\{\left(\mathrm{p}_{21.5}+\mathrm{p}_{21.5,16}+\mathrm{p}_{24} \mathrm{p}_{41.17}\right)\right.$ $\left.\left(1-\mathrm{p}_{33.10^{-}} \mathrm{p}_{93} \mathrm{p}_{39}\right)-\mathrm{p}_{13.8} \mathrm{p}_{32.7}\left\{\left(\mathrm{p}_{21.5}+\mathrm{p}_{21.5,16}+\mathrm{p}_{24} \mathrm{p}_{41.17}\right)\right\}\right]+\mu_{1}^{\prime}$ $\left[\left(\mathrm{p}_{01}\right)\left\{\quad\left(1-\mathrm{p}_{22.12-} \mathrm{p}_{22.12,15-} \mathrm{p}_{24} \mathrm{p}_{42.19}\right) \quad\left(1-\mathrm{p}_{33.10^{-}} \mathrm{p}_{93} \mathrm{p}_{39}\right) \quad-\right.\right.$ $\left.\mathrm{p}_{32.7}\left(\mathrm{p}_{23.11}+\mathrm{p}_{23.11,14}+\mathrm{p}_{24} \mathrm{p}_{43.18}\right)\right\}+\mathrm{p}_{02}\left\{\left(\mathrm{p}_{21.5}+\mathrm{p}_{21.5,16}+\mathrm{p}_{24} \mathrm{p}_{41.17}\right)\right.$ $\left.\left(1-\mathrm{p}_{33.10^{-}} \mathrm{p}_{93} \mathrm{p}_{39}\right)+\mathrm{p}_{03} \mathrm{p}_{32.7}\left\{\left(\mathrm{p}_{21.5}+\mathrm{p}_{21.5,16}+\mathrm{p}_{24} \mathrm{p}_{41.17}\right)\right\}\right]+\left(\mu_{2}^{\prime}+\right.$ $\left.\mathrm{p}_{24 \mu_{4}^{\prime}}\right)\left[\mathrm{p}_{32.7}\left\{\mathrm{p}_{01} \mathrm{p}_{13.8}+\mathrm{p}_{03}\left(1-\mathrm{p}_{11.13}\right)\right\}+\left(1-\mathrm{p}_{33.10^{-}} \mathrm{p}_{93} \mathrm{p}_{39}\right)\left\{\mathrm{p}_{01}\right.\right.$ $\left.\left.\mathrm{p}_{12.6}+\quad\left(1-\mathrm{p}_{11.13}\right) \mathrm{p}_{02}\right\}\right]+\quad\left(\mu_{3}^{\prime}+\mathrm{p}_{39} \mu_{9}^{\prime}\right) \quad\left[\mathrm{p}_{01}\left\{\mathrm{p}_{12.6}\left(\mathrm{p}_{23.11}+\right.\right.\right.$

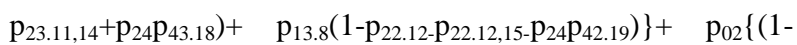
$\left.\mathrm{p}_{11.13}\right) \quad\left(\mathrm{p}_{23.11}+\quad \mathrm{p}_{23.11,14}+\mathrm{p}_{24} \mathrm{p}_{43.18}\right)+\quad \mathrm{p}_{13.8}\left(\mathrm{p}_{21.5}+\right.$

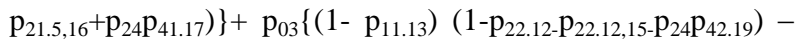
$\left.\left.\mathrm{p}_{12.6}\left(\mathrm{p}_{21.5}+\mathrm{p}_{21.5,16}+\mathrm{p}_{24} \mathrm{p}_{41.17}\right)\right\}\right]$

\subsection{Busy Period of the Server}

Let $B_{i}^{P}(t), B_{i}^{R}(t) \quad B_{i}^{S}(t)$ and $B_{i}^{H R p}(t)$ be the probabilities that the server is busy in Preventive maintenance of the system, repairing the unit due to hardware failure, replacement of the software and hardware components at an instant ' $\mathrm{t}$ ' given that the system entered state $\mathrm{i}$ at $\mathrm{t}=0$. The recursive relations for $B_{i}^{P}(t), B_{i}^{R}(t), B_{i}^{S}(t)$ and $B_{i}^{H R p}(t)$ are as follows:

$B_{i}^{p}(t)=W_{i}(t)+\sum_{j} q_{i, j}^{(n)}(t) \odot B_{j}^{p}(t)$

$B_{i}^{R}(t)=W_{i}(t)+\sum_{j} q_{i, j}^{(n)}(t) @ B_{j}^{R}(t)$

$B_{i}^{S}(t)=W_{i}(t)+\sum_{j} q_{i, j}^{(n)}(t) \odot B_{j}^{S}(t)$

$B_{i}^{H R p}(t)=W_{i}(t)+\sum_{j} q_{i, j}^{(n)}(t) \odot B_{j}^{H R p}(t)$

Where $j$ is any successive regenerative state to which the regenerative state $i$ can transit through $\mathrm{n} \geq 1$ (natural number) transitions. $\mathrm{W}_{\mathrm{i}}(\mathrm{t})$ be the probability that the server is busy in state $S_{i}$ due to preventive maintenance, hardware and software failure up to time $\mathrm{t}$ without making any transition to any other regenerative state or returning to the same via one or more non-regenerative states and so

$W_{1}=e^{-\left(a \lambda_{1}+b \lambda_{2}+\alpha_{0}\right) t} \bar{F}(t)+\left(\alpha_{0} e^{-\left(a \lambda_{1}+b \lambda_{2}+\alpha_{0}\right) t}(1) \overline{\mathrm{F}}(t)+\right.$

$\left(a \lambda_{1} e^{-\left(a \lambda_{1}+b \lambda_{2}+\alpha_{0}\right) t}(\mathrm{C}) \bar{F}(t)+\left(b \lambda_{2} e^{-\left(a \lambda_{1}+b \lambda_{2}+\alpha_{0}\right) t}\right.\right.$ C 1$) \bar{F}(t)$

$W_{9}=\bar{F}(t)$

$W_{2}=e^{-\left(a \lambda_{1}+b \lambda_{2}+\alpha_{0}+\beta_{0}\right) t} \bar{G}(t)+\left(\alpha_{0} e^{-\left(a \lambda_{1}+b \lambda_{2}+\alpha_{0}+\beta_{0}\right) t} \subset 1\right) \overline{\mathrm{G}}(t)+$

$\left(a \lambda_{1} e^{-\left(a \lambda_{1}+b \lambda_{2}+\alpha_{0}+\beta_{0}\right) t}(1) \bar{G}(t)+\left(b \lambda_{2} e^{-\left(a \lambda_{1}+b \lambda_{2}+\alpha_{0}+\beta_{0}\right) t} \subset 1\right) \bar{G}(t)\right.$

$W_{3}=e^{-\left(a \lambda_{1}+b \lambda_{2}+\alpha_{0}\right) t} \bar{H}(t)+\left(\alpha_{0} e^{-\left(a \lambda_{1}+b \lambda_{2}+\alpha_{0}\right) t}\right) \overline{\mathrm{H}}(t)$

$+\left(a \lambda_{1} e^{-\left(a \lambda_{1}+b \lambda_{2}+\alpha_{0}\right) t} \subseteq 1\right) \bar{H}(t)+\left(b \lambda_{2} e^{-\left(a \lambda_{1}+b \lambda_{2}+\alpha_{0}\right) t} \subseteq 1\right) \bar{H}(t)$

$\cdot W_{4}=e^{-\left(a \lambda_{1}+b \lambda_{2}+\alpha_{0}\right) t} \bar{M}(t)+\left(\alpha_{0} e^{-\left(a \lambda_{1}+b \lambda_{2}+\alpha_{0}\right) t} \odot 1\right) \overline{\mathrm{M}}(t)$
$+\left(a \lambda_{1} e^{-\left(a \lambda_{1}+b \lambda_{2}+\alpha_{0}\right) t}(\mathrm{C}) \bar{M}(t)+\left(b \lambda_{2} e^{-\left(a \lambda_{1}+b \lambda_{2}+\alpha_{0}\right) t}(\mathrm{C}) \bar{M}(t)\right.\right.$

Taking LT of above relations (12) and solving for $B_{i}^{P}(t)$ $B_{i}^{R}(t) \quad B_{i}^{S}(t)$ and $\quad B_{i}^{H R p}(t)$ the time for which server is busy due to $\mathrm{PM}, \mathrm{h} / \mathrm{w}$ repair and $\mathrm{h} / \mathrm{w}$ and $\mathrm{s} / \mathrm{w}$ replacements respectively is given by

$B_{0}^{H}=\lim _{s \rightarrow 0} s B_{0}^{* H}(s) \quad=\frac{N_{3}^{H}}{D_{2}} \quad, \quad B_{0}^{S}=\lim _{s \rightarrow 0} s B_{0}^{* S}(s)=\frac{N_{3}^{S}}{D_{2}}$, $B_{0}^{R}=\lim _{S \rightarrow 0} s B_{0}^{* R}(S)=\frac{N_{S}^{R}}{D_{2}}$

And $\quad B_{0}^{H R p}=\lim _{s \rightarrow 0} s B_{0}^{* H R p}(S)=\frac{N_{S}^{H R p}}{D_{2}}$ 


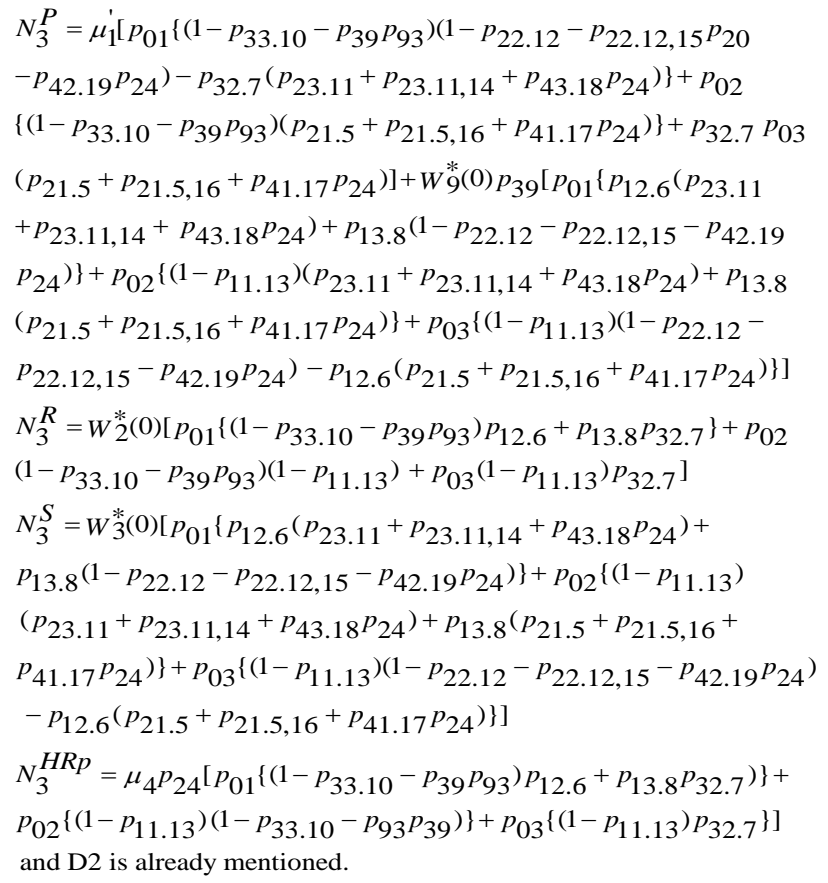

\subsection{Excepted Number of Replacements of the units}

Let $R_{i}^{H}(t)$ and $R_{i}^{S}(t)$ the expected number of replacements of the failed hardware and software components by the server in $(0, t]$ given that the system entered the regenerative state $\mathrm{i}$ at $\mathrm{t}=0$. The recursive relations for $R_{i}^{H}(t)$ and $R_{i}^{S}(t)$ are given as

$$
\begin{aligned}
& R_{i}^{H}(t)=\sum_{j} q_{i, j}^{(n)}(t) \circledast\left[\delta_{j}+R_{j}^{H}(t)\right] \\
& R_{i}^{S}(t)=\sum_{j} q_{i, j}^{(n)}(t) \circledast\left[\delta_{j}+R_{j}^{S}(t)\right]
\end{aligned}
$$

Where $\mathrm{j}$ is any regenerative state to which the given regenerative state $i$ transits and $\delta j=1$, if $j$ is the regenerative state where the server does job afresh, otherwise $\delta j=0$.

Taking LT of relations and, solving for $\tilde{R}_{0}^{H}(s)$ and $\tilde{R}_{0}^{S}(s)$. The expected numbers of replacements per unit time to the hardware and software failures are respectively of given by

$$
R_{0}^{H}(\infty)=\lim _{s \rightarrow 0} s \tilde{R}_{0}^{H}(s)=\frac{N_{4}^{H}}{D_{2}} \text { and } R_{0}^{S}(\infty)=\lim _{s \rightarrow 0} s \tilde{R}_{0}^{S}(s)=\frac{N_{4}^{S}}{D_{2}}
$$

Where $\mathrm{D}_{2}$ is already mentioned.

$$
\begin{aligned}
N_{4}^{H}= & \left(p_{23.11,14}+p_{21.5,16}+p_{22.12,15}+p_{24}\right)\left\{p _ { 0 1 } \left[\left(1-p_{33.10}-p_{93} p_{39}\right)\right.\right. \\
& \left.p_{12.6}+p_{13.8} p_{32.7}\right]+p_{02}\left[\left(1-p_{11.13}\right)\left(1-p_{33.10}-p_{39} p_{93}\right)\right]+p_{03} \\
& \left.\left(1-p_{11.13}\right) p_{32.7}\right\} \\
N_{4}^{S}= & \left(p_{30}+p_{32.7}+p_{33.10}\right)\left[p _ { 0 1 } \left\{p_{12.6}\left(p_{23.11}+p_{23.11,14}+p_{43.18} p_{24}\right)+\right.\right. \\
& \left.p_{13.8}\left(1-p_{22.12}-p_{22.12,15}-p_{42.19} p_{24}\right)\right\}+p_{02}\left\{( 1 - p _ { 1 1 . 1 3 } ) \left(p_{23.11}\right.\right. \\
+ & \left.\left.p_{23.11,14}+p_{43.18} p_{24}\right)+p_{13.8}\left(p_{21.5}+p_{21.5,16}+p_{41.17} p_{24}\right)\right\}+p_{03} \\
& \left\{\left(1-p_{11.13}\right)\left(1-p_{22.12}-p_{22.12,15}-p_{42.19} p_{24}\right)-p_{12.6}\left(p_{21.5}+\right.\right. \\
& \left.\left.\left.p_{21.5,16}+p_{41.17} p_{24}\right)\right\}\right]
\end{aligned}
$$

\subsection{Expected Number of Visits by the Server}

Let $\mathrm{N}_{\mathrm{i}}(\mathrm{t})$ be the expected number of visits by the server in $(0$, $\mathrm{t}$ ] given that the system entered the regenerative state $\mathrm{i}$ at $\mathrm{t}=$ 0 . The recursive relations for $\mathrm{N}_{\mathrm{i}}(\mathrm{t})$ are given as

$N_{i}(t)=\sum_{j} q_{i, j}^{(n)}(t) \circledast\left[\delta_{j}+N_{j}(t)\right]$

Where $\mathrm{j}$ is any regenerative state to which the given regenerative state $i$ transits and $\delta j=1$, if $j$ is the regenerative state where the server does job afresh, otherwise $\delta j=0$.

Taking LT of relation (20) and solving for $\tilde{N}_{0}(s)$. The expected number of visit per unit time by the server are given by

$$
N_{0}(\infty)=\lim _{s \rightarrow 0} s \tilde{N}_{0}(s)=\frac{N_{5}}{D_{2}}, \text { where }
$$

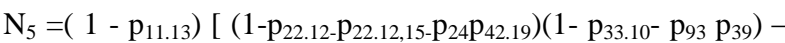
$\left.\mathrm{p}_{32.7}\left(\mathrm{p}_{23.11}+\mathrm{p}_{23.11,14}+\mathrm{p}_{24} \mathrm{p}_{43.18}\right)\right]-\mathrm{p}_{12.6}\left(1-\mathrm{p}_{33.10^{-}} \mathrm{p}_{93} \mathrm{p}_{39}\right)\left(\mathrm{p}_{21.5}+\right.$ $\left.\mathrm{p}_{21.5,16}+\mathrm{p}_{24} \mathrm{p}_{41.17}\right)-\mathrm{p}_{13.8} \mathrm{p}_{32.7}\left(\mathrm{p}_{21.5}+\mathrm{p}_{21.5,16}+\mathrm{p}_{24} \mathrm{p}_{41.17}\right)$

\section{Ecohómic Analysis}

The profit incurred to the system model in steady state can be obtained as

$P=K 0 A 0-K 1 B_{0}^{P}-K 2 B_{0}^{R}-K 3 B_{0}^{S}-K 4 B_{0}^{H R p}-K 5 R_{0}^{H}-K 6 R_{0}^{S}-K 7 N_{0}$

$\mathrm{K}_{0}=$ Revenue per unit up-time of the system

$\mathrm{K}_{1}=$ Cost per unit time for which server is busy due preventive maintenance

$\mathrm{K}_{2}=$ Cost per unit time for which server is busy due to hardware failure ,

$\mathrm{K}_{3}=$ Cost per unit replacement of the failed software component

$\mathrm{K}_{4}=$ Cost per unit replacement of the failed hardware component , $\mathrm{K}_{5}=$ Cost per unit replacement of the failed hardware $\mathrm{K}_{6}=$. Cost per unit replacement of the failed software $\mathrm{K}_{7}=$ Cost per unit visit by the server 


\section{CONCLUSION}

In the present study, the numerical results considering a particular case $g(t)=\theta e^{-\theta t}, h(t)=\beta e^{-\beta t}$, $f(t)=\alpha e^{-\alpha t}$ and $m(t)=\gamma e^{-x}$ are obtained for some reliability and economic measures of a computer system of two identical units having $\mathrm{h} / \mathrm{w}$ and $\mathrm{s} / \mathrm{w}$ components. The graphs for mean time to system failure (MTSF), availability and profit are drawn with respect to preventive maintenance rate $(\alpha)$ for fixed values of other parameters as shown respectively in figs. 2 to 4. From these figures, it is revealed that MTSF, Availability and profit increase with the increase of PM rate $(\alpha)$ and repair rate $(\theta)$ of the hardware components. But the value of these measures increase with the increase of maximum operation time $\left(\alpha_{0}\right)$. Again if we increase the value of maximum constant rate of repair time $\left(\beta_{0}\right)$, the value of MTSF, availability and profit are increase. Thus on the basis on the results obtained for a particular case, it is suggested that the reliability and profit of a system in which chances of $\mathrm{h} / \mathrm{w}$ failure are high can be improved by

(i) Reducing the repair time of the $\mathrm{h} / \mathrm{w}$ components as well as conducting PM of the units after a prespecific period of time.

(ii) Making replacement of the hardware components by new one in case repair time is too long.

(iii) Making replacement of s/w components by new one.

\section{ACKNOWLEDGMENTS}

The authors are thankful to the Department of Science \&Technology, New Delhi, India for Providing Financial Assistance for this research work under INSPIRE Fellowship.

\section{REFERENCES}

[1] Friedman, M. A. and Tran, P.1992 Reliability Techniques for Combined Hardware/Software Systems,
Proc. Of Annual Reliability and Maintability Symposiym, pp.290-293.

[2] Welke, S. R.; Labib, S. W. and Ahmed, A. M.1995 Reliability Modeling of Hardware/ Software System, IEEE Transactions on Reliability, Vol.44, No.3, pp.413418.

[3] Singh, S. K. and Agrafiotis, G. K.1995. Stochastic Analysis of a Two- Unit Cold Standby System Subject to Maximum Operation and Repair Time, Microelectron.Reliab., Vol.35,No.12,pp.1489-1493.

[4] Malik, S. C. and Jyoti Anand 2010. Reliability and Economic Analysis of a Computer System with Independent Hardware and Software Failures, Bulletin of Pure and Applied Sciences, Vol.29 E (Math. \& Stat.), No. 1, pp.141-153.

[5] Malik, S. C. and Nandal, P.2010. Cost- Analysis of Stochastic Models with Priority to Repair Over Preventive Maintenance Subject to Maximum Operation Time, Edited Book, Learning Manual on Modeling, Optimization and Their Applications, Excel India Publishers, pp.165-178.

[6] Malik, S. C. and Ashish Kumar 2011. Profit Analysis of a Computer System with Priority to Software Replacement over Hardware Repair Subject to Maximum Operation and Repair Times, International Journal of Engineering Science \& Technology, Vol.3, No. 10, pp. 7452- 7468

[7] Anand, Jyoti and Malik,S.C.2011. Reliability Modeling of a Computer System with Priority for Replacement at software Failure over Repair Activities at Hardware Failure. International Journal of Statistics and Systems, Vol.6(3), pp. 315-325.

[8] Anand, Jyoti; Malik. S.C. and Kumar Ashish. 2011. Cost-Analysis Of A Computer System With Hardware Repair Subject To Inspection And Arbitrary Distributions For Hardware and Software Replacement. International Journal of Engg. Science and Technology (IJEST), Vol.3 (9), pp.7191-7204. 
Fig.1 State Transition Diagram

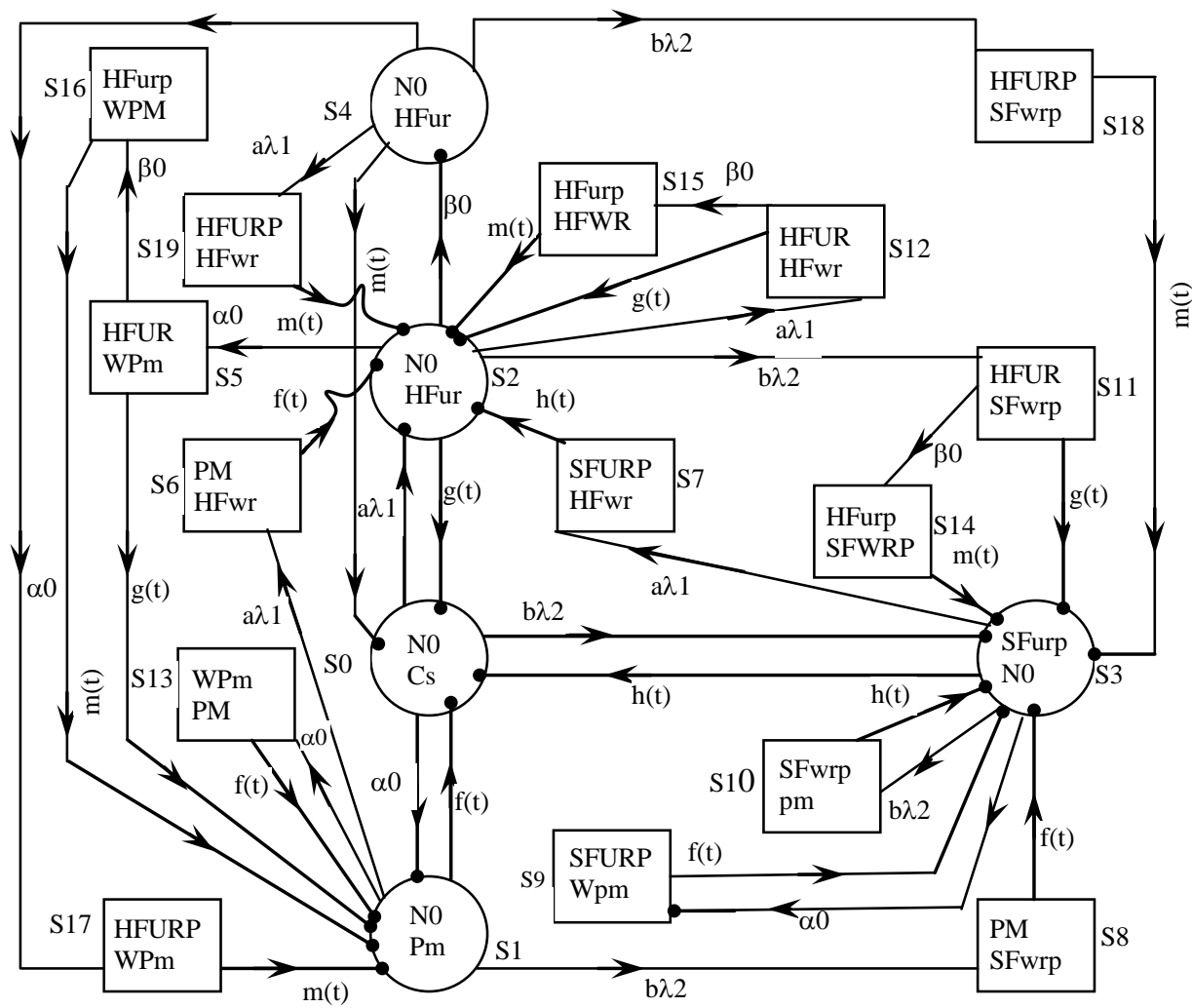

$\bigcirc \Rightarrow$ Operative State

$\Rightarrow \quad$ Failed State , $\quad \Rightarrow \quad$ Regenerative Point

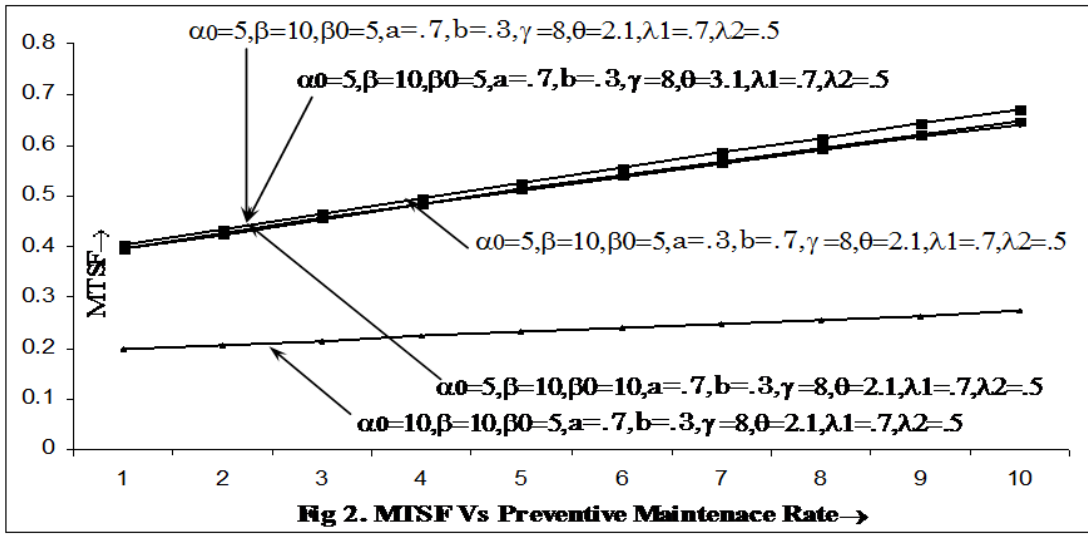



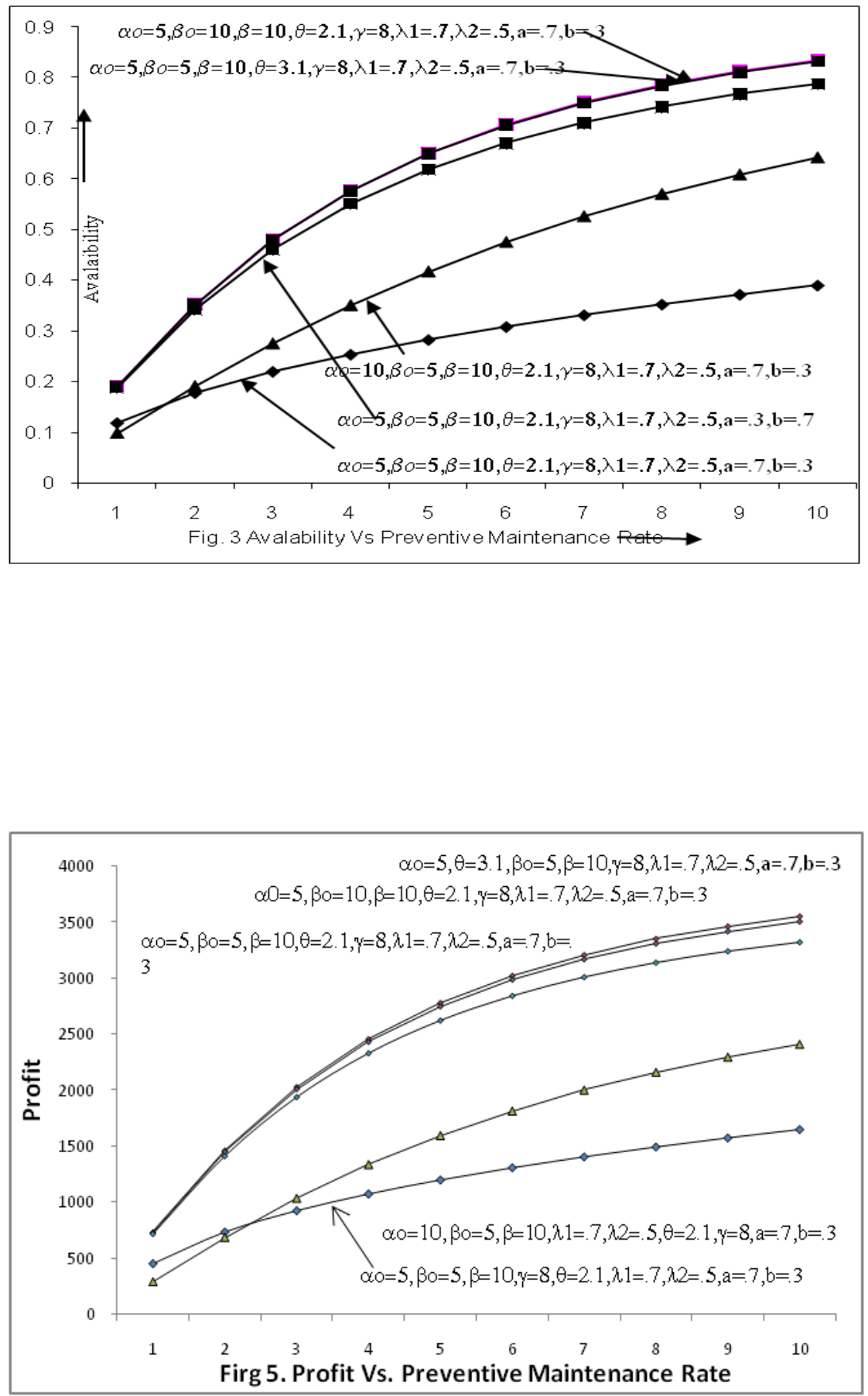